\title{
HST Observations of the HH 47 and HH 111 Stellar Jets $^{1}$
}

\author{
Patrick Hartigan \\ Space Physics and Astronomy Dept, Rice University, Houston TX \\ 77251-1892
}

\begin{abstract}
The superb spatial resolution made possible by the WFPC2 imager on the Hubble Space Telescope has opened a new era in the study of shocks in stellar jets. This paper summarizes the results of imaging surveys of two of the brightest and best resolved stellar jets, HH 111 and HH 47 . The new HST images show that the jets appear quite different in the $\mathrm{H} \alpha$ and [S II] lines; $\mathrm{H} \alpha$ concentrates in distinctive filaments while [S II] tends to be clumpier and more widespread. Filamentary $\mathrm{H} \alpha$ emission should trace the location of the shock fronts if the preshock gas is neutral, because a significant fraction of the incident $\mathrm{H}$ gas becomes excited collisionally before becoming ionized in moderate velocity shocks.

Both $\mathrm{HH} 111$ and $\mathrm{HH} 47$ exhibit multiple bow shocks along the channel of the jet, and in some cases we observe the classical double shock structure of a bow shock/Mach disk expected within the working surface of a jet. The jet material is considerably denser than that of the ambient gas. Ejection angles vary a few degrees with time, giving some jets a 'wiggling' appearance. However, motion along the jet appears to be radial; there is no convincing evidence for azimuthal motion in the radial velocity data. Multiple bow shocks observed along the jets probably arise from unsteady accretion of a protostellar disk onto the central object.
\end{abstract}

\section{Introduction}

Herbig-Haro objects were first identified as emission line objects in dark clouds over 40 years ago (Haro 1950; Herbig 1951). Though they have lower excitation than supernovae remnants, $\mathrm{HH}$ objects also exhibit a variety of ionization states, which led to their characterization as shocks (Schwartz 1975). High proper motions confirmed the shocked nature of the emission and showed that material was ejected supersonically from the young star (Cudworth \& Herbig 1979; Herbig \& Jones 1981). Broad line profiles of the brightest objects are consistent with material being splattered in a bow shock geometry (Schwartz 1978; Hartmann \& Raymond 1984; Hartigan et al. 1987).

Within the last decade it has become apparent that the brightest HH objects are typically bow shocks located along stellar jets (Reipurth \& Heathcote 1993;

\footnotetext{
${ }^{1}$ Based on observations made with the NASA/ESA Hubble Space Telescope, obtained at the Space Telescope Science Institute, which is operated by the Association of Universities for Research in Astronomy, Inc., under NASA contract NAS5-26555.
} 
Ray \& Mundt 1993). The jets themselves are visible as strings of low-excitation knots which emanate from infrared sources thought to mark the location of young stars surrounded by accretion disks. Indeed, accretion appears to be the ultimate energy source for jets - while the mechanical power in the jet often exceeds the photospheric luminosity, it is always less than the accretion luminosity. Comparison of mass accretion and mass outflow rates suggest that jets remove anywhere from $1 \%$ to $10 \%$ of the mass accreted onto the star (Hartigan et al. 1995). The mass accretion rates, inferred from the luminosity of the boundary layers, vary by orders of magnitude during FU Ori eruptions, and these eruptions probably manifest themselves later as bow shocks in the jet (Hartmann et al. 1993). In this way, the bow shocks in stellar jets record the history of major events of disk accretion onto the protostar. Jets may also play an important role in regulating the angular momenta of young stars. Surprisingly, young stars that accrete rotate more slowly than their non-accreting counterparts, as if the additional angular momentum acquired by accretion were removed in some way by an outflow (Edwards et al. 1993; Bouvier et al. 1993).

In addition to providing key information about the star formation process, stellar jets also indicate how highly supersonic flows interact with their surroundings under conditions of low density, high velocity, and large size scales that cannot be simulated in terrestrial laboratories or modeled completely with existing codes. Because stellar jets radiate and the shock fronts are resolved spatially, it is possible to observe the radial velocities, line ratios, and proper motion of the emission lines along the flow. These data provide electron densities and temperatures at each velocity for each point in the flow, a powerful data set to compare with theoretical models (e.g. Morse et al. 1992; 1994). Observations of time variability and proper motions yield equally useful constraints.

This paper briefly summarizes the results of imaging and spectroscopic studies of the bright stellar jets HH 47 and HH 111. Because of space limitations, it is not possible to describe each jet in detail. For more complete discussions of the new images we refer the reader to Heathcote et al. 1996 (HH 47), and Reipurth et al. 1997 (HH 111). The physics behind the interpretation of the data are presented in $\S 2$, while new results for various portions of the jets appear in $\S 3, \S 4$, and $\S 5$. The primary conclusions are summarized in $\S 6$.

\section{H $\alpha$ Emission and the Location of Shocks}

Ideally, we would like to observe the density and velocity at each point within the jet as a function of time to see how the jet evolves as it interacts with itself and its surroundings. In particular, because the flow is controlled by the location, morphology and strength of the shock fronts, we need to define these as precisely as possible. Unfortunately, only atoms that have recently passed through a shock and are in the process of cooling will radiate emission lines that are detectable in narrow band images. Any jet gas with temperatures $\lesssim 10^{3} \mathrm{~K}$ will not radiate substantially in the standard nebular lines of $\mathrm{H} \alpha$, [S II], [N II], [O I], [O II], or [O III] even if the gas is quite dense. It is important to remember these facts when comparing images with numerical simulations.

In most cases the gas ahead of the shock also does not radiate strongly enough to be observed, so it is not possible to observe a discontinuity in the 
density across the shock directly. Even when the gas does radiate, we can only measure the electron density, and not the total density directly from line ratios like [S II] $\lambda 6716 /[\mathrm{S} \mathrm{II}] \lambda 6731$. The distinction between $\mathrm{N}_{e}$ and $\mathrm{N}_{T O T}$ is an important one, as the ionization fraction in stellar jets is typically only a few percent (Hartigan et al. 1995).

In spite of the above difficulties, nature has provided us with an extremely powerful, yet simple means to identify shock fronts in neutral gas. When neutral hydrogen enters a shock there is a chance that the atom will become excited by collisions to $n=3$ before it is ionized. Such an atom may then radiate an $\mathrm{H} \alpha$ photon, so that Balmer emission should trace the precise location of the shocks in the flow. This process will only operate if the cross section for collisional excitation is comparable to that for ionization over the incident velocities of interest.

As Figure 1 demonstrates, the cross sections $\sigma$ and rate coefficients $\sigma \mathrm{v}$ for excitation are indeed comparable with that for ionization over several orders of magnitude in the incident kinetic energy of the atom. At first glance this behavior may seem strange, as the electron has a continuum of states it may populate for ionization, and only a single state for excitation. However, in the dipole approximation the collisional cross section for both excitation and ionization is proportional to the amplitude of the matrix element $\left|\left\langle\phi_{i}|e r| \phi_{f}\right\rangle\right|^{2}$, where $\phi_{i}$ and $\phi_{f}$ are the initial and final states, respectively. The cross section for ionization is dominated by states that have low kinetic energies for the final particle, and the matrix element for these is comparable to matrix elements for bound-bound transitions.

For Cases $\mathrm{A}$ and $\mathrm{B}$ recombination, the probability $\mathrm{P}_{H \alpha}$ that an atom emits an $\mathrm{H} \alpha$ photon before being ionized is

$$
\begin{aligned}
& P_{H \alpha(A)}=\left(\frac{\sigma_{13}}{\sigma_{i}}\right)\left(\frac{A_{32}}{A_{31}+A_{32}}\right) \\
& \quad+\left(\frac{\sigma_{14}}{\sigma_{i}}\right)\left(\frac{A_{32}}{A_{31}+A_{32}}\right)\left(\frac{A_{43}}{A_{41}+A_{42}+A_{43}}\right)+\ldots \sim 0.10
\end{aligned}
$$

and

$$
P_{H \alpha(B)}=\left(\frac{\sigma_{13}}{\sigma_{i}}\right)+\left(\frac{\sigma_{14}}{\sigma_{i}}\right)\left(\frac{A_{43}}{A_{43}+A_{42}}\right)+\ldots \sim 0.21
$$

where $\sigma_{1 n}$ is the cross section for collisions from level $1 \rightarrow n$, and $\sigma_{i}$ is the cross section for ionization from level 1.

Because charge exchange processes alter the velocity distribution of the neutrals significantly (Chevalier \& Raymond 1978), computing appropriate averages of the cross sections over velocity is somewhat involved. If we approximate the average by the ratio of rate coefficients over the energies of interest, we find $\left\langle\sigma_{13} / \sigma_{i}\right\rangle \sim 0.21$ and $\left\langle\sigma_{14} / \sigma_{i}\right\rangle \sim 0.06$. Taking into account that an atom which has a probability $P$ of emitting one $\mathrm{H} \alpha$ photon before being ionized has a probability $P^{k}$ of emitting $\mathrm{k} \mathrm{H} \alpha$ photons before being ionized, we find that the average number of photons $\mathrm{N}_{H \alpha}$ emitted per atom is $\mathrm{P} /(1-\mathrm{P}) \sim 0.11$ for Case $\mathrm{A}$, and 0.32 for Case B. These numbers agree well with the detailed calculations of 


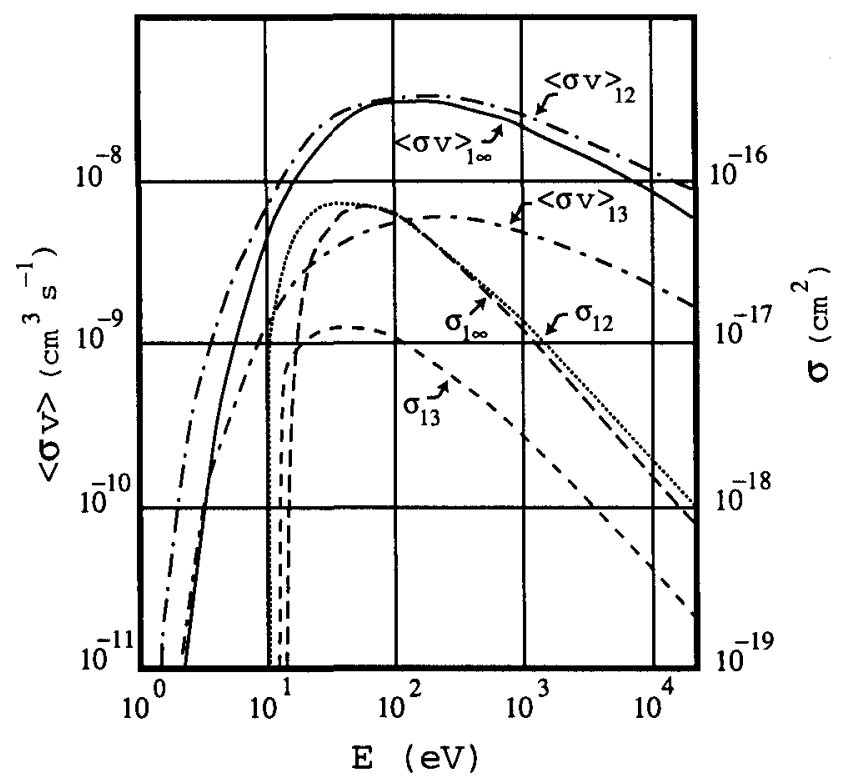

Figure 1. Cross sections and rate coefficients for collisional excitation and ionization of hydrogen plotted as a function of energy (adapted from Janev et al. 1987). The solid and dot-dashed curves $\left(\langle\sigma v\rangle_{1 i}\right.$, where $i=2,3, \infty)$ show the rate coefficients for collisional excitation and ionization for $n=1 \rightarrow i$ by thermal electrons at temperature $\mathrm{T}$ $=\mathrm{E} / \mathrm{k}$. The dashed and dotted curves are cross sections and use the ordinate at right. For shock velocities of interest $\left(30-300 \mathrm{~km} \mathrm{~s}^{-1} ; 1.8\right.$ $-180 \mathrm{eV}$ ) the cross sections for collisional excitation are comparable to the cross section for collisional ionization. Collisional excitation and ionization by protons and charge exchange to excited states become important (more than a $10 \%$ correction to $\langle\sigma v\rangle$ ) when $\mathrm{E}\rangle 10^{3} \mathrm{eV}$.

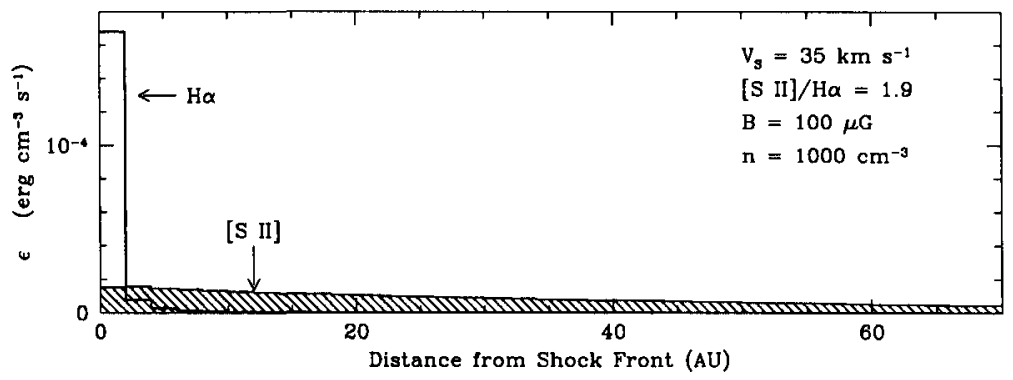

Figure 2. A plot of the surface brightness of $\mathrm{H} \alpha$ and [S II] line emission behind a low velocity shock (adapted from Heathcote et al. 1996). The $\mathrm{H} \alpha$ emission arises primarily from collisional excitation at the shock front. 
Chevalier et al. (1980), who find $\mathrm{N}_{H \alpha}=0.09$ and 0.43 , respectively, for cases $\mathrm{A}$ and $\mathrm{B}$.

We conclude from this exercise that Balmer emission should arise from the shock fronts whenever the preshock gas has a substantial neutral component. Balmer emission also occurs downstream from the front as the gas cools and recombines, but Figure 2 shows that Balmer emission from the front appears brighter because it radiates within a small region. Hence, any sharp, arc-like Balmer emission is likely to mark the location of shock fronts in the flow. We will use this logic to interpret the images discussed in the following sections.

\section{Bow Shocks and Mach Disks}

When two fluids collide supersonically a shock is driven into each fluid. In jets the forward shock corresponds to a bow shock that accelerates the ambient gas, while the reverse shock, known as a 'Mach disk' decelerates material in in the jet. If the jet shuts off for a period of time, the Mach disk will disappear while the bow shock continues to plow through the ambient material until it sweeps up enough mass and slows down.

There are several prominent bow shocks in both the HH 47 and HH 111 jets. In HH 47A we observe clearly both the bow shock and the Mach disk via the Balmer emission (Figures 3 and 4). These observations show that the jet must have a strong neutral component. The situation for the bright bow shocks ' $\mathrm{L}$ ' and ' $\mathrm{V}$ ' in the HH 111 jet is less obvious (Reipurth et al. 1997). Two bow shocks may be in the process of merging in $\mathrm{HH} 111 \mathrm{~L}$ and the Mach disk is weak or absent in HH 111V. In all cases the [S II] emission separates from the $\mathrm{H} \alpha$, as expected for gas that cools behind a shock (Figure 2).

\section{Balmer Arcs}

The Balmer emission in the HH 47 jet is much more filamentary than the [S II], outlines weak shocks that propagate laterally from the jet into the surrounding medium (Figure 5). Hence, jets sweep up material by pushing it aside rather than by entraining matter in a turbulent manner (i.e. 'prompt' entrainment dominates; DeYoung 1986). The material which surrounds the jet must be mostly neutral to generate the strong $\mathrm{H} \alpha$ emission at the shock without a bright cooling zone of [S II] trailing behind. No high excitation lines are observed near the arcs, so the effective shock velocity must be only $\sim 30-50 \mathrm{~km} \mathrm{~s}^{-1}$. The jet moves at several hundred $\mathrm{km} \mathrm{s}^{-1}$ from the star, so these low shock velocities imply that the gas just outside the jet must also move outward at nearly the speed of the jet. This gas was probably pushed aside by wakes of previous bow shocks.

The HH 111 jet (Figure 6) also shows a series of full and one-sided bow shocks along the jet, and these shocks resemble the $\mathrm{HH} 47$ arcs as they extend into the surrounding medium in the $\mathrm{H} \alpha$ image. The flux of $\mathrm{H} \alpha$ drops by almost an order of magnitude outside of the jet, so there must be a corresponding drop in the preshock density there (because the $\mathrm{H} \alpha$ flux is proportional to the flux of atoms through the shock). We conclude that the gas to the sides of the jet is fast, tenuous, and mostly neutral. 


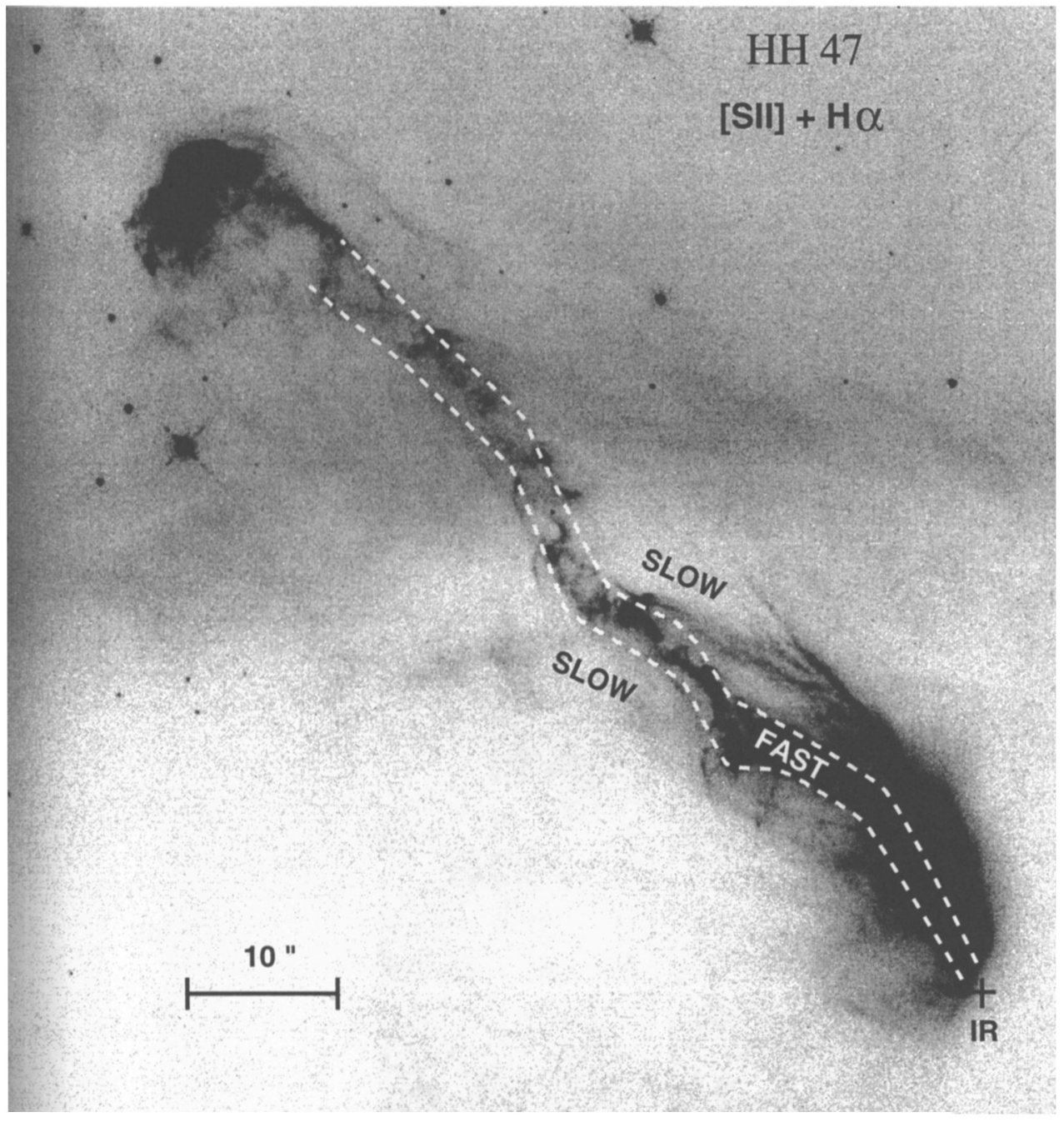

Figure 3. A composite of the $\mathrm{HH} 47$ jet showing the variation of the ejection angle of the flow (from Heathcote et al. 1996). The infrared source is labeled with a ' + '. The bright bow-shaped object in the upper left portion of the image is HH 47A (Figure 4). The 'fast' and 'slow' velocities marked in the figure are discussed in the text. 

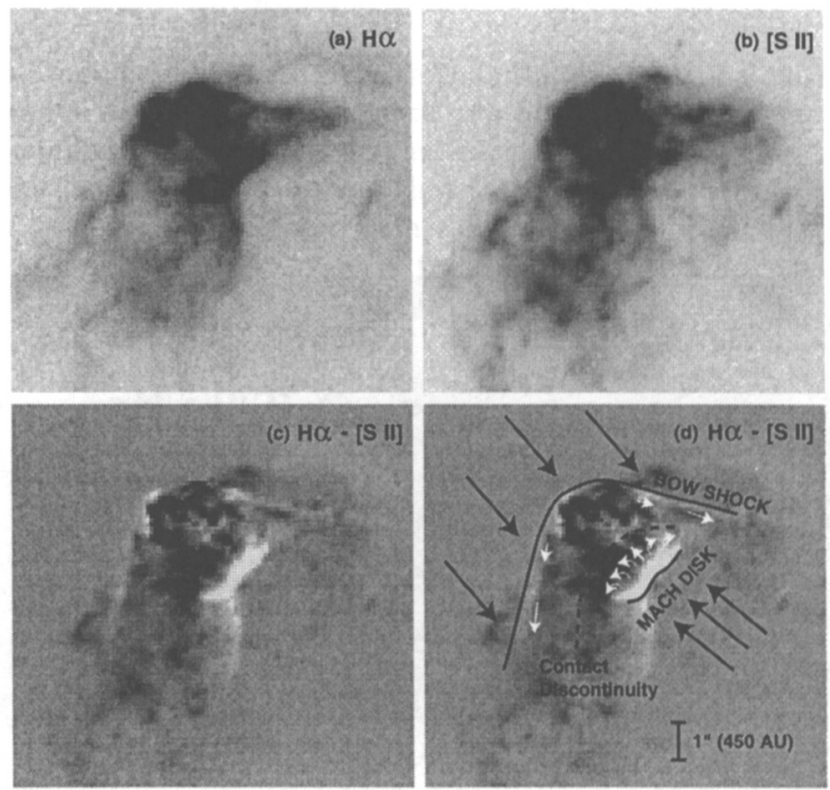

Figure 4. The bow shock and Mach disk of HH 47A (from Heathcote et al. 1996). The $\mathrm{H} \alpha$ emission clearly defines the location of both shocks, with the [S II] dominating the cooling zone between them.
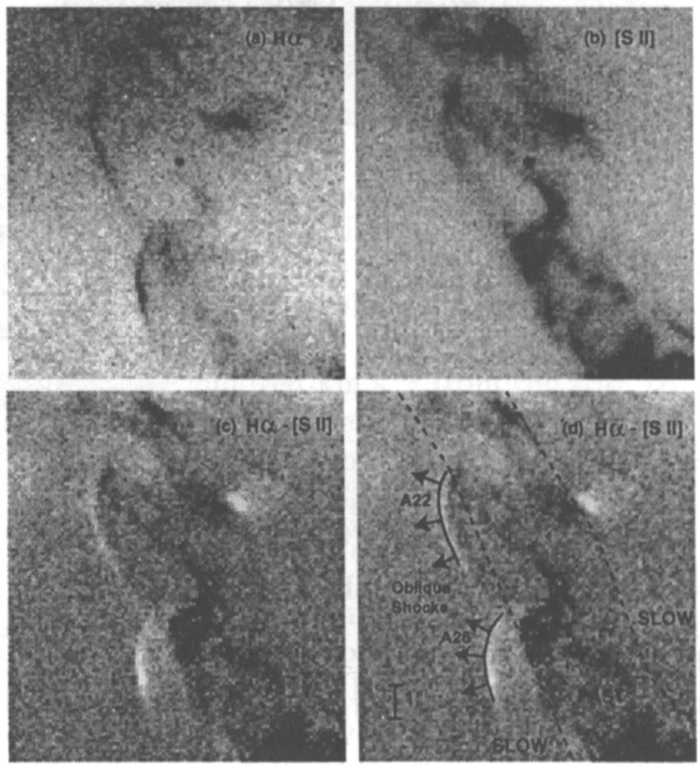

Figure 5. The Balmer arcs in $\mathrm{HH} 47$, show how the jet entrains the surrounding gas (from Heathcote et al. 1996). 

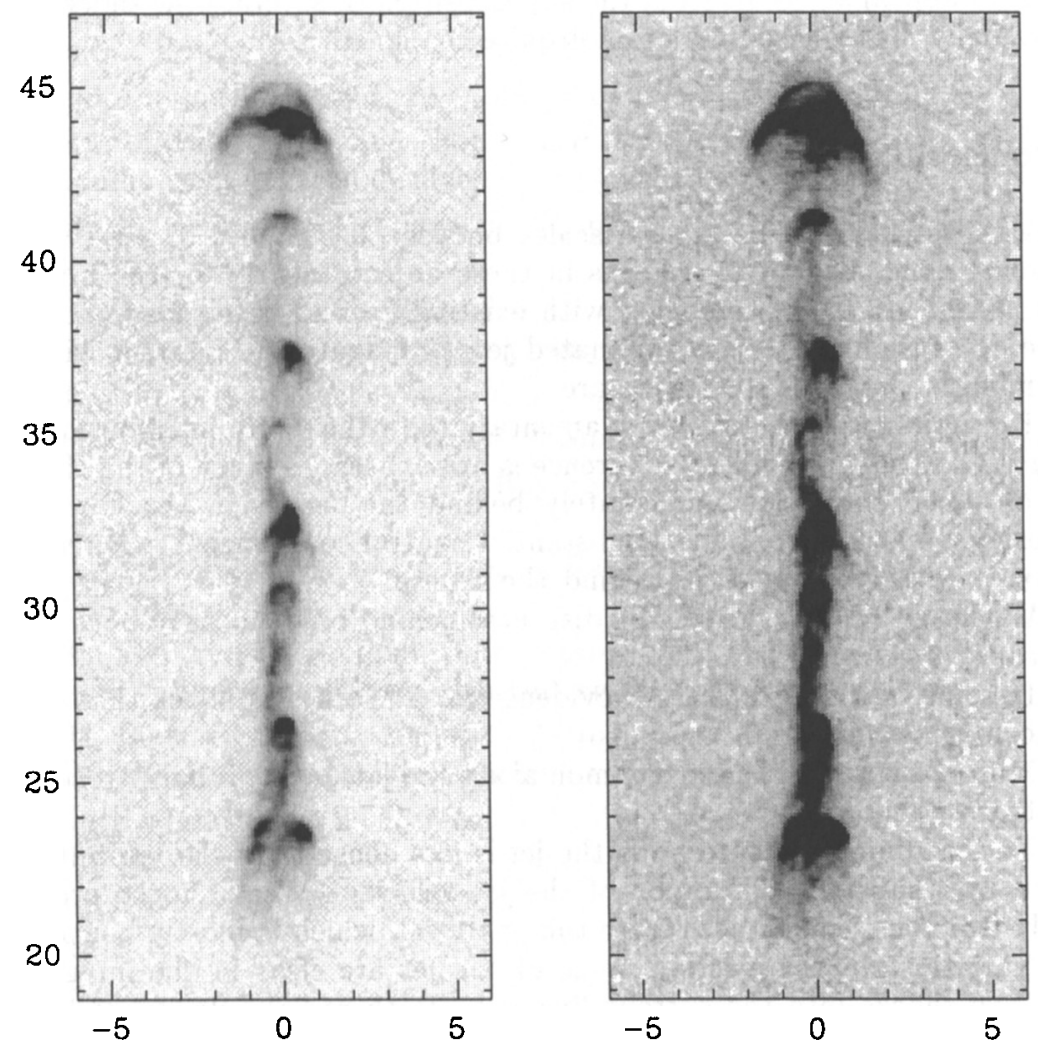

Figure 6. An $\mathrm{H} \alpha$ image of the $\mathrm{HH} 111$ jet shown at two different contrast levels. The jet consists of multiple bow shocks, some of which resemble the arcs in $\mathrm{HH}$ 47. (from Reipurth et al. 1997). The axes are labeled in units of arcseconds offset from the exciting source. One arcsecond corresponds to $450 \mathrm{AU}$ for this object. 


\section{Shocks along the Jet}

Figure 6 shows that the shocks along jets, while sometimes complex, often resolve into a series of bow shocks. The easiest way to explain these observations is if the jet varies in velocity so that the fastest material overruns the slower gas and generates bow shocks. Figure 3 shows that the ejection angle of the jet varies with time, and this variation must produce some of the shocks that we observe. In $\mathrm{HH} 47$ the fastest material moves along the axis of the jet, consistent with a picture where the jet moves away from the star radially, and drives material ahead of it and laterally via shocks. There is as yet no convincing evidence for any spiraling motion of the jet around its axis. The 'wiggles' observed in the images appear to result from the superposition of several bow shocks which move radially, but whose axes are not quite coaligned.

\section{Conclusions}

By imaging stellar jets on spatial scales between $0.1^{\prime \prime}$ and $1.0^{\prime \prime}$ HST resolves the cooling zones behind the shocks in these objects clearly for the first time. The HST images, when combined with existing ground-based kinematic data, provide new insights into how collimated jets propagate and interact with their surroundings. Our primary results are:

- $\mathrm{H} \alpha$ emission appears more filamentary than the [S II] emission in $\mathrm{HH} 47$ and $\mathrm{HH}$ 111. This morphology difference is arises because much of the $\mathrm{H} \alpha$ arises from collisional excitation immediately behind the shocks in the flow (where the preshock material contains a substantial neutral component), whereas [S II] emits only in the cooling zones behind the shocks.

- We clearly resolve the cooling distances behind bow shocks in both HH 111 and $\mathrm{HH} 47$.

- In some bow shocks there is evidence for a Mach disk shock that decelerates incoming jet material.

- Multiple bow shocks are common along the jets, and probably result from a nonsteady outflow.

- The medium that surrounds the jet is less dense than the jet, but moves outward at a substantial fraction of the jet velocity. Weak shocks propagate laterally from the jet and accelerate this material, which is mostly neutral.

- Variations in the ejection angle of the jet are clear in the images, and these drive shocks into the surrounding gas. Both radial velocity and proper motion data indicate that the gas moves away from the exciting source radially; there is no convincing evidence for any spiraling motions.

- The images show no unambiguous evidence for a 'focusing shock' that might collimate the jet.

The next step in the study of these jets will be to reobserve them with HST and compare the proper motions with those predicted by numerical simulations. Our team (Reipurth et al. 1997) has been awarded imaging time in the next HST cycle to address this issue.

Acknowledgments. The author would like to thank J. Weisheit for discussions concerning atomic cross sections. 


\section{References}

Bouvier, J., Cabrit, S., Fenandez M., Martin, E., Matthews, J. 1993, A\&AS 101, 485.

Chevalier, R.A., Kirschner, R.P., Raymond, J.C. 1980, ApJ 235, 186.

Chevalier, R.A., Raymond, J.C. 1978, ApJ 225, L27.

Cudworth, K.M., and Herbig, G.H. 1979, AJ 84, 548.

DeYoung, D. 1986, ApJ 307, 62.

Edwards, S., et al. 1993, AJ 106, 372.

Haro, G. 1950, AJ 55, 72.

Hartigan, P., Raymond, J.C., \& Hartmann, L. 1987, ApJ 316, 323.

Hartigan, P., Edwards, S., \& Ghandour, L. 1995, ApJ 452, 736.

Hartmann, L., and Raymond, J.C. 1984, ApJ 276, 560.

Hartmann, L., Kenyon, S., and Hartigan, P. Protostars and Planets III, Levy \& Lunine eds., (Tucson: University of Arizona Press), p. 497.

Heathcote, S., Morse, J., Hartigan, P., Reipurth, B., Schwartz, R.D., Bally, J., \& Stone, J. 1996, AJ 112, 1141.

Herbig, G.H. 1951, ApJ 113, 697.

Herbig, G.H., and Jones, B.F. 1981, AJ 86, 1232.

Janev, R.K., Langer, W.D., Evans, K.Jr., and Post, D.E.Jr. 1987, Elementary Processes in Hydrogen-Helium Plasmas, (Berlin: Springer-Verlag).

Morse, J.A., Hartigan, P., Cecil, G., Raymond, J.C., \& Heathcote, S. 1992, ApJ $399,231$.

Morse, J.A., Hartigan, P., Heathcote, S., Raymond, J.C., \& Cecil, G. 1994, ApJ $425,738$.

Ray, T.P., and Mundt, R. 1993, Astrophysical Jets, Burgarella, Livio, \& O'Dea eds., (Cambridge: Cambridge University Press), p145.

Reipurth, B. \& Heathcote, S. 1993, Astrophysical Jets, Burgarella, Livio, \& O'Dea eds., (Cambridge: Cambridge University Press), p35.

Reipurth, B., Heathcote, S., Hartigan, P., Morse, J., \& Bally, J. 1997, in preparation.

Schwartz, R.D. 1975, ApJ 195, 631.

Schwartz, R.D. 1978, ApJ 223, 884. 